\title{
Do Cancer Stem Cells have an Immunomodulatory Role Different from the Bulk of Tumor Cells?
}

\section{Hazem Ghebeh* and Monther Al-Alwan}

Stem Cell \& Tissue Re-engineering Program, King Faisal Specialist Hospital and Research Centre, Saudi Arabia

\begin{abstract}
Cancer is one of the leading causes of death worldwide. In normal settings, the immune system is responsible for clearing cancer cells from our body. However, many patients develop immune tolerance to tumor cells through upregulation of immune regulatory molecules, release of immune suppressive factors in the tumor microenvironment and/or recruitment of regulatory/suppressive cells that impede the function of other fully activated effector immune cells. In spite of significant progress that have been achieved in increasing our understanding in this field, it is unknown whether all tumor cells exert similar inhibitory effect on the immune system or only specific subset(s) of tumor cells possess this feature.
\end{abstract}

There is accumulating evidence that cancer is originated and sustained by a small population of cells called "Cancer Stem Cells (CSCs)". These cells share many characteristics of the normal stem cells including the selfrenewing ability. Thus, it is possible that they also have the immune privilege properties of normal stem cells. At least this has been shown to be the case for two types of cancers: melanoma and glioma.

In this report we will review the role of CSCs in the creation of immune suppressive microenvironment which finally leads to tumor escape from the immune system surveillance.

Keywords: Cancer stem cells; Glioma; Melanoma; Epithelial-tomesenchymal-transition; Immune response

\section{Introduction and Background}

The incidence of cancer is increasing worldwide and in spite of the significant advances achieved in cancer treatment, the mortality resulting from this disease is high. Cancer is a complex disease where transformed cells acquire various biological features that are essential to the initiation and maintenance of the disease. These features include: enhanced proliferative signaling, evasion of growth suppressors, increased resistance to death, enabled replicative immortality, induced angiogenesis and activated invasion and metastasis, reprogrammed energy metabolism and evaded immune system [1].

In most cancer cases, it is believed that increased mortality happens due to residual cancer cells remaining after surgery and/or induction of chemotherapy [2]. This residual population leads to disease relapse and/or tumor metastasis to other organs [3]. Successful cure for cancer is largely dependent on effective immune system that can detect and combat these cells to clear them from the system in addition to other established treatments like surgery and chemotherapy. In many cancer cases there are several signs of immune system activation against cancer cells, nevertheless some tumors keep growing [4]. This immune tolerance to tumor cells is attributed to the upregulation of regulatory molecules and/or release of suppressive factors in the tumor microenvironment [5]. However, the tumor tissue component that contributes directly or indirectly to the upregulation of regulatory molecules or the formation of the immune suppressive environment is not well-identified.

Normal tissues from different organs are maintained by "adult stem cells", which are cells with unique ability of continuous self-renewal and differentiation to specialized cell types [6]. Although they represent a small fraction of their respective tissue, stem cells are responsible for maintaining our cells homeostasis by replacing dead, injured and malfunctioning cells. Their proliferation is partially regulated by their supportive microenvironment cells usually termed as "niche" [7].
In cancer, a small subset of the tumor acquires some of the stem cell features and thus named as "cancer stem cells" (CSCs) [8]. Although still not definitive, there is mounting evidence that a hierarchy exist, where CSCs differentiate into cancer non-stem cells and form the bulk of the tumor [9]. Evidence show that in experimental settings, CSCs are solely responsible for the generation of tumors when implanted in immunocompromised mice, while their differentiated counterpart cells are not [10]. Importantly, recent supporting evidence confirms such a hierarchy even in unperturbed tumor model using genetic tracking systems [11-13]. While CSCs only represent a small fraction of malignant cells, they have been shown to be resistant to radiotherapy [14] and chemotherapy [15] leading to relapse of cancer and metastasis [3], which is believed to be the main cause of increased cancer mortality. In this review we will focus on one of the hallmarks of cancer i.e. immune escape of cancer cells.

\section{Immunogenicity of Stem Cells}

The immune system is designed to recognize cells expressing non-self antigens and clear them from the system. This process is tightly regulated and dependent on the activation of antigen-specific $\mathrm{T}$ cells by professional antigen presenting cells such as dendritic cells, macrophages or B cells, with dendritic cells being the most potent and widely believed to be the only one that can prime naïve $\mathrm{T}$ cells [16]. Activation of $\mathrm{T}$ cells is a multistep process that is triggered

*Corresponding author: Hazem Ghebeh, Stem Cell \& Tissue Re-engineering Program, King Faisal Specialist Hospital and Research Centre, Saudi Arabia, E-mail: hghebeh@kfshrc.edu.sa

Received April 02, 2013; Accepted April 29, 2013; Published May 10, 2013

Citation: Ghebeh H, Al-Alwan M (2013) Do Cancer Stem Cells have an Immunomodulatory Role Different from the Bulk of Tumor Cells? J Carcinogene Mutagene S14: 003. doi:10.4172/2157-2518.S14-003

Copyright: (C) 2013 Ghebeh $\mathrm{H}$, et al. This is an open-access article distributed under the terms of the Creative Commons Attribution License, which permits unrestricted use, distribution, and reproduction in any medium, provided the original author and source are credited. 
when their $\mathrm{T}$ cell receptors recognize cognate peptide-MHC class II complex on the surface of professional antigen presenting cells [17]. While this step provides critical signal for the initiation of the immune response, a second signal is required to ensure productive activation of $\mathrm{T}$ cells that leads to the clearance of the antigen-baring cells whether pathogen-infected or cancer cells. The second signal is provided by co-stimulatory molecules such as CD80, CD86, CD40 or adhesion molecules such as LFA-1 and ICAM-1 [18-21]. In contrast, there are negative co-stimulatory molecules like B7-H1 (also called PD-L1 and CD274) that suppress the immune response, thereby modulate the immune response [18]. In addition, it has been well documented that the presence of some soluble factors or certain cell types during the antigen presentation step influence the immune response either by generating the wrong type of immune response or by suppressing the immune response [5]. For example, presence of TGF- $\beta 1$ during antigen presentation is known to induce regulatory $\mathrm{T}$ cells (a subset of T-cell population characterized phenotypically by being CD4+CD25+FOXP3+ and ability to inhibit effector T-cells function) [19]. Altogether, above show that deregulation of the tightly controlled immune response may result in immune escape of cancer cells.

\section{Immunogenicity of Embryonic Stem Cells}

Recent studies showed that stem cells exhibit cytotoxic and phagocytic activities [20,21] and have a machinery for antigen presentation contrary to the previous concept that they are immunologically null [22]. This notion is further supported by presence of immune reactions to stem cells that are implanted for the sake of cell replacement in degenerative disease animal models. Nevertheless, stem cells from different organs and hierarchy still show many signs of immune-privilege when compared with differentiated cells. For example, embryonic stem cells have many signs of immune privilege [23] and are less susceptible to rejection than adult cells [24]. Furthermore, their immunogenicity is tremendously increased after differentiation $[25,26]$. Recently, embryonic cells were shown to be immunogenic, but their inherent immune privilege property promote the induction of tolerance by reducing the number of professional antigen presenting cells and increased expression of soluble factors that favor the generation of regulatory $\mathrm{T}$ cells [27]. In addition, cord blood stem cells have been shown to be immune privileged through the expression of B7-H1 (a negative co-stimulatory molecule that inhibit T-cell activation) and it is ability to induce regulatory T-cells [28]. Interestingly, reprogramming adult cells by merely introduction of four transcriptional factors to generate pluripotent stem cells (iPSC) did not have such immune privilege [29], suggesting the presence of other genetic differences between embryonic stem cells and iPSCs.

\section{Immunogenicity of Mesenchymal Stem Cells}

Mesenchymal stem cells (MSCs) are a relatively rare population of cells in the bone marrow that are able to differentiate into bone, fat and cartilage. Although they are not yet established as true stem cells, i.e. if they are able to maintain a whole tissue in vivo from a single cell, they are clearly multi-potent both in vitro and in vivo [30]. The immunomodulatory role of MSCs in vitro is well-established which is reviewed by Abdi et al. [4]. MSCs inhibit T-cell proliferation [31], abrogate lysis by CD8+ cytotoxic T-cells [32] and increase the proportion of FOXP3+ T-reg [33]. The mechanism involved in MSC mediated immunomodulation is partially explained by their expression of the negative co-stimulatory molecule B7-H1 [34], and their ability to produce hepatic growth factor (HGF), IL-10, and/or TGF- $\beta 1$. These immune-modulatory properties of stem cells made them an attractive therapeutic potential and explain their wide use in many clinical trials to treat variety of disorders like: severe graft-versus-host disease, tissue repair and treatment of some autoimmune disorders [30].

Unfortunately, in cancer tissues, MSCs support the growth of tumors and suppress the immune system. For example MSCs produce CXCL7 which in turn induce the synthesis of IL8, a strong inducer of CSC self-renewal capability [35]. In addition, IL-1 $\alpha$ which is present in the tumor environment [36] makes MSC promote the growth of cancer cells [37]. On the other hand, MSCs, mainly through their ability to recruit T-reg, can suppress the migration and proliferation of peripheral blood mononuclear cells (PBMC) and inhibit NK and cytotoxic T- cell (CTL) functions [38].

\section{The Immune-Suppressive Effect of Cancer Stem Cells (CSCs)}

CSCs possess similar features to normal stem cells in their ability of inducing immune modulation. Unfortunately, possession of these features by CSCs contributes to their escape from the immune system recognition and thus failure of the treatment and tumor relapse. Therefore, there is growing interest in understanding the mechanisms that regulate CSC immune modulatory properties in order to develop more effective therapy that can eradicate these cells.

There are many signs that tumors in general show signs of immune tolerance. This is manifested by their ability to attract CD4+CD25+ FOXP3+ regulatory T-cells [39], their expression of B7-H1 [40], their lack of co-stimulatory molecules like CD80 and CD86 (positive costimulatory molecules that are required for optimal T-cell activation) and their occasional lack of MHC class I molecules [41]. In our previous work we have shown that immune inhibitory molecules like B7-H1 is expressed on tumor cells, B7-H1 is expressed on tumor infiltrating lymphocytes, and FOXP3+ regulatory T-cells are abundant in the tumor microenvironment in a group of breast cancer patients. The expression of these molecules correlated with tumors that were estrogen receptor negative, high histological grade and large tumor size $[42,43]$.

In addition, we found a significant correlation between the expression the negative co-stimulatory molecule $(\mathrm{B} 7-\mathrm{H} 1)$ and the actin-bundling protein (fascin) [44]. The latter is important for normal mature dendritic cells function particularly in regulating their morphology, binding with antigen-specific $\mathrm{T}$ cells and generation of a productive immune response [45-47]. Interestingly, induction of fascin expression in many types of tumor is always associated with bad prognosis and shorter survival [48]. Although there is no direct evidence yet for a role of fascin in the immune escape of breast cancer cells, our study demonstrated, in breast cancer model, a novel role of this protein in regulating metastasis associated genes. Strikingly, Chen et al. [49] used a xenograft mouse model to demonstrate that the selective targeting of fascin by a compound named "migrastatin" inhibits breast cancer metastasis into the lung.

CSCs are the only cells that are able to re-establish a tumor with its heterogeneity when injected in an immunocompromised host [10]. However, when the immune system is further compromised a large fraction of the tumor cells can form tumors and not just CSCs. This has been shown by Quintana et al where up to $28 \%$ of tumor cells could form a tumor when injected in severely compromised IL-2 receptor deficient NOD/SCID mice (NOD/SCID/IL-2R $\gamma^{-/ 2}$ ) and not with original NOD/SCID mice [50]. This suggests a degree of interaction between CSCs and host immune system as only CSCs can overcome a 
certain degree of leaky immune response present in NOD/SCID mice and not the bulk of the tumor. Altogether, these studies suggest that it is possible that CSCs among the cancer cells are immune privileged which protect the tumor from the immune system recognition at least initially at the early stages of tumor development. At least this has been shown to be the case in two type of tumors i.e. glioma and melanoma [51].

\section{CSCs in Melanoma}

Melanoma is one of the main causes of death related to skin cancer and it is particularly resistant to therapy [52]. Melanoma CSCs have been identified as ATP-binding cassette sub-family $\mathrm{B}$ member 5 (ABCB5) positive [53]. Specifically, ABCB5+ melanoma CSCs had significantly lower expression of MHC class I compared with the bulk of melanoma cells. In addition, the negative co-stimulatory receptor B7-H1 was preferentially expressed by ABCB5+ CSCs compared with the bulk $\left(\mathrm{ABCB}^{\text {neg }}\right)$ of melanoma cells. These observations suggest a lower ability of CSCs to induce an immune response compared with the bulk of the tumor [54]. Indeed, ABCB5+ melanoma CSCs blocked mitogen-stimulated $\mathrm{PBMC}$ proliferation by $93 \%$ and this inhibition was significantly greater than that exerted by $\mathrm{ABCB} 5^{\text {neg }}$ bulk population. These findings provided strong evidence for the immune suppression property of melanoma CSCs and make it an attractive target for therapeutic intervention.

\section{CSCs in Glioma}

Glioma is considered one of the most aggressive malignancies of the brain and central nervous system [55,56]. Like many other type of cancer, the glioma microenvironment become immune suppressive where primed CD8+ cytotoxic T-cells become inhibited or induced to become apoptotic and thus cannot execute their function $[57,58]$. In addition, there is increased recruitment of other immune suppressive cells like FOXP3 + regulatory T-cells [59]. Importantly, recent work has shown that this suppressive effect is specially exerted by glioma CSCs. This small subset of glioma cells, characterized by being CD133+ glioma cells (i.e., glioma CSCs), specially was able to inhibit T-cell proliferation and induce T-cell apoptosis via cell-to-cell mediated fashion and B7$\mathrm{H} 1$ appears to play a central role in this process [60]. Indeed, inhibition of T-cell activation by the presence of CSCs to the co-culture was partially reversed by the addition of B7-H1 blocking antibody [60]. This is not to mention that glioma CSCs produced cytokines like TGF1, CCL-2 and prostaglandin E2, which either prompt the propagation of FOXP3+ regulatory T cells, skewed the immune response into Th2 (not effective against cancer) or employed a global immunosuppressive effect. Importantly, glioma CSCs were shown to specifically produce galactin-3 leading to T-cell apoptosis [60].

In addition to the direct immunosuppressive effect of glioma CSCs, they recruit regulatory/suppressive cells which in turn modulate the immune response. For example, glioma CSCs induce FOXP3 regulatory T-cells partially involving B7-H1 molecule [60]. Furthermore, glioma CSCs, and not the bulk of the tumor, induce the differentiation of immune suppressive/tumor supportive type of macrophages (M2) from monocytes which is believed to be mediated through secreted macrophage inhibitor cytokine 1 (MIC-1) [61]. Interestingly, many

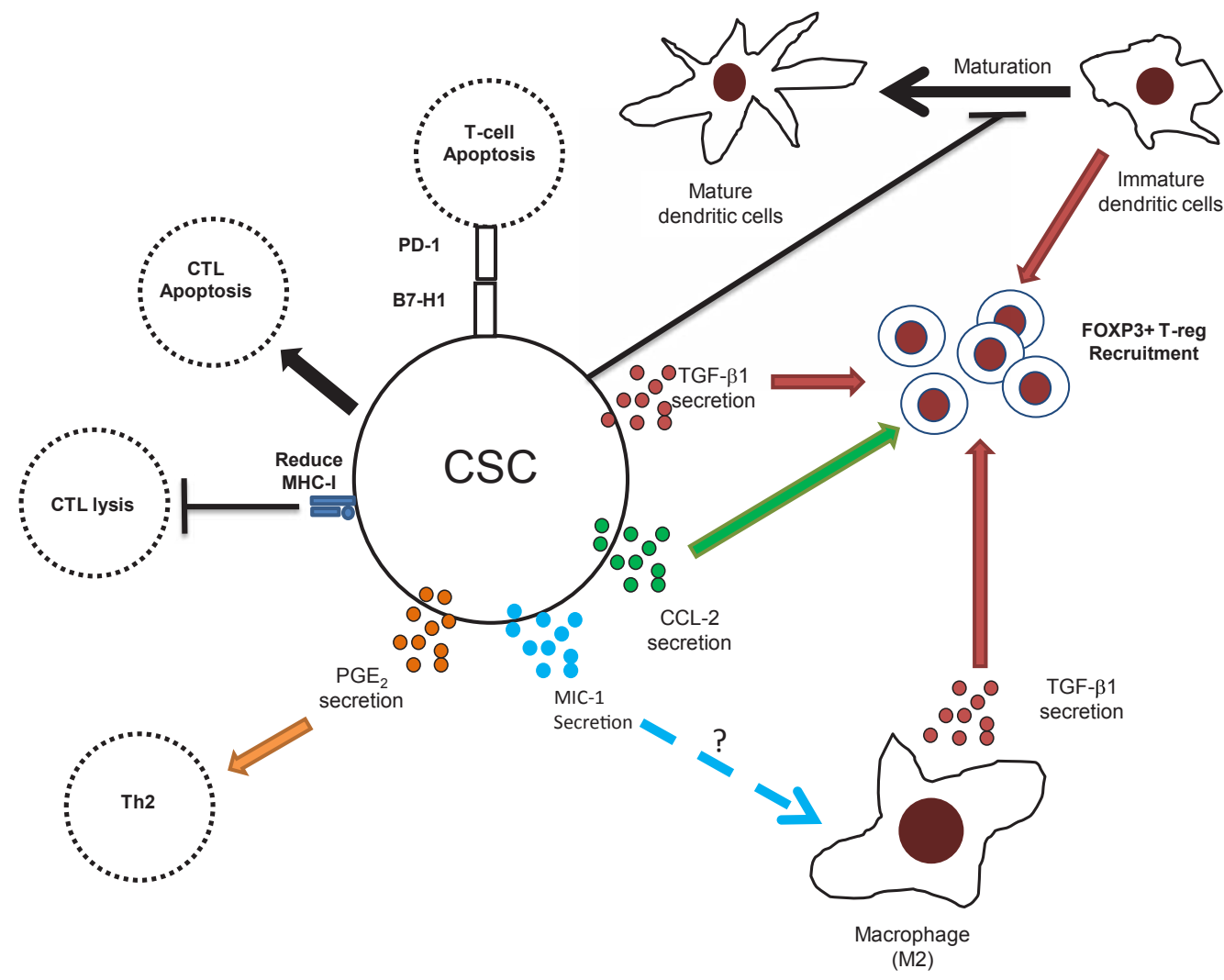

Figure 1: Immunomodulatory role of cancer stem cells (CSCs). Schematic diagram that summarizes the immunosuppressive role of CSC. PGE2= Prostaglandins $\mathrm{E} 2, \mathrm{MIC}=$ macrophage inhibitor cytokine 1 , TGF- $\beta 1=$ Tumor growth factor beta $1 \mathrm{CCL} 2=, \mathrm{T}$-reg= Regulatory T-cells. Straight line $(\underset{\longrightarrow}{\longrightarrow}$ indicates evidence supported effect while a dotted line (- indicates a possible/plausible mechanism. 
of the suppressive effect of glioma CSC could be reversed specifically by signal transducers and Activators and Transcription 3 (STAT3) pathway [62]. Altogether, these factors contribute directly or indirectly to the immunosuppressive property of glioma CSCs and further validate the notion that CSCs are an important subset of cancer cells responsible for the tumor escape from immune recognition.

\section{The Role of Cancer Induced EMT and Tumor Immune Escape}

Breast cancer is one of the most common type of tumor in women [63]. While recent years have witnessed significant advances in the treatment of breast cancer, breast cancer mortality remained high mainly due to residual cancer cells that are therapy resistant and are able to re-grow and metastasize [3]. Many scientists believe that this subset of cells is able to do so via a process called Epithelial to Mesenchymal Transition (EMT) whereby epithelial cells lose their cell-to-cell junctions and gain features of mesenchymal cells like higher migration, and invasion. This process is paralleled with a change in some of the cell surface markers like CD44, CD24, vimentin and E-cadherin. Mesenchymal cells have a CD $44^{\text {high }} / \mathrm{CD} 24^{\text {low }}$, vimentin+ and E-cadherin ${ }^{\text {neg }}$ phenotype, while epithelial cells normally have $\mathrm{CD} 24^{\text {high }}$, vimentin ${ }^{\text {neg }}$ and E-Cadherin+ phenotype. During the EMT process epithelial cells acquire the $\mathrm{CD} 44^{\text {high }} / \mathrm{CD} 24^{\text {low }}$ phenotype, upregulate vimentin and down-regulate E-Cadherin. Importantly, there is an established link between this process and the gain of stem cell functions in breast cancer [64]. This includes the ability to grow in anchorage independent conditions and to reestablish tumors in mice models even when a very small number of cells are transplanted. Interestingly, when breast cancer cells were induced to go through EMT process under the influence of the transcriptional factor Snail, an EMT inducing gene, they promoted the escape of breast cancer cells from T-cell mediated lysis by CD8+ cytotoxic T cells [65]. This further supports the role of CSCs in the immune escape of breast cancer cells. This effect is not limited to breast cancer but was also found to be relevant to melanoma. Melanoma cells induced to go though EMT under the influence of Snail generated CD4+FOXP3+ T-reg and impaired the maturation of dendritic cells both in vivo and in vitro [66]. Collectively, the above data support that EMT, a process that enrich for CSCs, exhibit immunomodulatory property.

\section{Conclusion}

Despite the controversies regarding the existence of CSCs and their exact phenotype, mounting evidence is accumulating to support the importance of this unique subset of tumor cells. There are several evidences that CSCs employ immunosuppressive effect, thereby evading the immune recognition. Different CSCs may employ different mechanisms to confer this immunosuppressive effect either directly by regulating the expression of molecules such as the negative immune regulator molecule like $\mathrm{B} 7-\mathrm{H} 1$ or indirectly by influencing the tumor microenvironment to dictate the type of immune cell generated by favoring the induction or recruitment of FoxP3+ T-reg (Figure 1). These findings attract therapeutic intervention that involves targeting these CSCs and their immunosuppressive effect.

\section{Acknowledgements}

HG is supported by KFSH\&RC project (RAC\# 2080-045) and King Abdulaziz City for Science and Technology (KACST) project (AT-29-24). MA is supported by KFSH\&RC project (RAC\#2060-016).

\section{References}

1. Hanahan D, Weinberg RA (2011) Hallmarks of cancer: the next generation. Cell 144: 646-674.
2. Yu Y, Ramena G, Elble RC (2012) The role of cancer stem cells in relapse of solid tumors. Front Biosci (Elite Ed) 4: 1528-1541.

3. Nicolini A, Giardino R, Carpi A, Ferrari P, Anselmi L, et al. (2006) Metastatic breast cancer: an updating. Biomed Pharmacother 60: 548-556.

4. Abdi R, Fiorina P, Adra CN, Atkinson M, Sayegh MH (2008) Immunomodulation by mesenchymal stem cells: a potential therapeutic strategy for type 1 diabetes. Diabetes 57: 1759-1767.

5. Gajewski TF (2007) Failure at the effector phase: immune barriers at the leve of the melanoma tumor microenvironment. Clin Cancer Res 13: 5256-5261.

6. Al-Hajj M, Clarke MF (2004) Self-renewal and solid tumor stem cells. Oncogene 23: $7274-7282$

7. Bissell MJ, Labarge MA (2005) Context, tissue plasticity, and cancer: are tumor stem cells also regulated by the microenvironment? Cancer Cell 7: 17-23.

8. Gupta PB, Chaffer CL, Weinberg RA (2009) Cancer stem cells: mirage or reality? Nat Med 15: 1010-1012.

9. Gilbertson RJ, Graham TA (2012) Cancer: Resolving the stem-cell debate Nature 488: 462-463.

10. Visvader JE, Lindeman GJ (2008) Cancer stem cells in solid tumours: accumulating evidence and unresolved questions. Nat Rev Cancer 8: 755-768.

11. Chen J, Li Y, Yu TS, McKay RM, Burns DK, et al. (2012) A restricted cel population propagates glioblastoma growth after chemotherapy. Nature 488 522-526.

12. Driessens G, Beck B, Caauwe A, Simons BD, Blanpain C (2012) Defining the mode of tumour growth by clonal analysis. Nature 488: 527-530.

13. Schepers AG, Snippert $H J$, Stange DE, van den Born $M$, van $E s ~ J H$, et al. (2012) Lineage tracing reveals Lgr5+ stem cell activity in mouse intestinal adenomas. Science 337: 730-735.

14. Bao S, Wu Q, McLendon RE, Hao Y, Shi Q, et al. (2006) Glioma stem cells promote radioresistance by preferential activation of the DNA damage response. Nature 444: 756-760.

15. Frank NY, Margaryan A, Huang Y, Schatton T, Waaga-Gasser AM, et al (2005) ABCB5-mediated doxorubicin transport and chemoresistance in human malignant melanoma. Cancer Res 65: 4320-4333.

16. Metlay JP, Puré E, Steinman RM (1989) Distinct features of dendritic cells and anti-lg activated $B$ cells as stimulators of the primary mixed leukocyte reaction. J Exp Med 169: 239-254.

17. Steinman RM (1991) The dendritic cell system and its role in immunogenicity. Annu Rev Immunol 9: 271-296.

18. Francisco LM, Sage PT, Sharpe AH (2010) The PD-1 pathway in tolerance and autoimmunity. Immunol Rev 236: 219-242.

19. Zhao Y, Huang Z, Qi M, Lazzarini P, Mazzone T (2007) Immune regulation of T lymphocyte by a newly characterized human umbilical cord blood stem cell. Immunol Lett 108: 78-87.

20. Law S, Begum B, Chaudhuri S (2003) Pluripotent bone marrow cells in leukemic mice elicit enhanced immune reactivity following sheep erythrocyte administration in-vivo. A possible S-LFA3 interactive immunotherapy. J Exp Clin Cancer Res 22: 213-221.

21. Law S, Maiti D, Palit A, Majumder D, Basu K, et al. (2001) Facilitation of functional compartmentalization of bone marrow cells in leukemic mice by biological response modifiers: an immunotherapeutic approach. Immunol Lett 76: 145-152.

22. Sujata L, Chaudhuri S (2008) Stem cell niche, the microenvironment and immunological crosstalk. Cell Mol Immunol 5: 107-112.

23. Li L, Baroja ML, Majumdar A, Chadwick K, Rouleau A, et al. (2004) Human embryonic stem cells possess immune-privileged properties. Stem Cells 22 448-456.

24. Drukker $M$, Katchman $H$, Katz G, Even-Tov Friedman S, Shezen $E$, et al. (2006) Human embryonic stem cells and their differentiated derivatives are less susceptible to immune rejection than adult cells. Stem Cells 24: 221-229.

25. Swijnenburg RJ, Tanaka M, Vogel H, Baker J, Kofidis T, et al. (2005) Embryonic stem cell immunogenicity increases upon differentiation after transplantation into ischemic myocardium. Circulation 112: I166-172. 
Citation: Ghebeh H, Al-Alwan M (2013) Do Cancer Stem Cells have an Immunomodulatory Role Different from the Bulk of Tumor Cells? J Carcinogene Mutagene S14: 003. doi:10.4172/2157-2518.S14-003

26. van der Bogt KE, Swijnenburg RJ, Cao F, Wu JC (2006) Molecular imaging of human embryonic stem cells: keeping an eye on differentiation, tumorigenicity and immunogenicity. Cell Cycle 5: 2748-2752.

27. Robertson NJ, Brook FA, Gardner RL, Cobbold SP, Waldmann H, et al. (2007) Embryonic stem cell-derived tissues are immunogenic but their inherent immune privilege promotes the induction of tolerance. Proc Natl Acad Sci U S A 104: 20920-20925.

28. Habicht A, Dada S, Jurewicz M, Fife BT, Yagita H, et al. (2007) A link between PDL1 and T regulatory cells in fetomaternal tolerance. J Immunol 179: 5211 5219.

29. Zhao T, Zhang ZN, Rong Z, Xu Y (2011) Immunogenicity of induced pluripotent stem cells. Nature 474: 212-215.

30. Le Blanc K, Ringdén O (2007) Immunomodulation by mesenchymal stem cells and clinical experience. J Intern Med 262: 509-525

31. Bartholomew A, Sturgeon C, Siatskas M, Ferrer K, McIntosh K, et al. (2002) Mesenchymal stem cells suppress lymphocyte proliferation in vitro and prolong skin graft survival in vivo. Exp Hematol 30: 42-48.

32. Angoulvant D, Clerc A, Benchalal S, Galambrun C, Farre A, et al. (2004) Human mesenchymal stem cells suppress induction of cytotoxic response to alloantigens. Biorheology 41: 469-476.

33. Selmani Z, Naji A, Zidi I, Favier B, Gaiffe E, et al. (2008) Human leukocyte antigen-G5 secretion by human mesenchymal stem cells is required to suppress T lymphocyte and natural killer function and to induce CD4+CD25highFOXP3+ regulatory T cells. Stem Cells 26: 212-222.

34. Augello A, Tasso R, Negrini SM, Amateis A, Indiveri F, et al. (2005) Bone marrow mesenchymal progenitor cells inhibit lymphocyte proliferation by activation of the programmed death 1 pathway. Eur J Immunol 35: 1482-1490.

35. Liu S, Ginestier C, Ou SJ, Clouthier SG, Patel SH, et al. (2011) Breast cancer stem cells are regulated by mesenchymal stem cells through cytokine networks. Cancer Res 71: 614-624.

36. Apte RN, Voronov E (2002) Interleukin-1--a major pleiotropic cytokine in tumorhost interactions. Semin Cancer Biol 12: 277-290.

37. Cheng J, Li L, Liu Y, Wang Z, Zhu X, et al. (2012) Interleukin-1 ̂̂士 induces immunosuppression by mesenchymal stem cells promoting the growth of prostate cancer cells. Mol Med Rep 6: 955-960.

38. Patel SA, Meyer JR, Greco SJ, Corcoran KE, Bryan M, et al. (2010) Mesenchymal stem cells protect breast cancer cells through regulatory $T$ cells: role of mesenchymal stem cell-derived TGF-beta. J Immunol 184: 5885-5894.

39. Curiel TJ, Coukos G, Zou L, Alvarez X, Cheng P, et al. (2004) Specific recruitment of regulatory $T$ cells in ovarian carcinoma fosters immune privilege and predicts reduced survival. Nature Medicine 10: 942-949.

40. Zang X, Allison JP (2007) The B7 family and cancer therapy: costimulation and coinhibition. Clin Cancer Res 13: 5271-5279.

41. Dermime S, Armstrong A, Hawkins RE, Stern PL (2002) Cancer vaccines and immunotherapy. Br Med Bull 62: 149-162.

42. Ghebeh H, Barhoush E, Tulbah A, Elkum N, Al-Tweigeri T, et al. (2008) FOXP3+ Tregs and B7-H1+/PD-1+ T lymphocytes co-infiltrate the tumor tissues of highrisk breast cancer patients: Implication for immunotherapy. BMC Cancer 8: 57 .

43. Ghebeh H, Tulbah A, Mohammed S, Elkum N, Bin Amer SM, et al. (2007) Expression of $\mathrm{B} 7-\mathrm{H} 1$ in breast cancer patients is strongly associated with high proliferative Ki-67-expressing tumor cells. Int J Cancer 121: 751-758.

44. Al-Alwan M, Olabi S, Ghebeh H, Barhoush E, Tulbah A, et al. (2011) Fascin is a key regulator of breast cancer invasion that acts via the modification of metastasis-associated molecules. PLoS One 6: e27339.

45. Al-Alwan MM, Rowden G, Lee TD, West KA (2001) The dendritic cell cytoskeleton is critical for the formation of the immunological synapse. J Immunol 166: 1452-1456.
46. Al-Alwan MM, Rowden G, Lee TD, West KA (2001) Fascin is involved in the antigen presentation activity of mature dendritic cells. J Immunol 166: 338-345.

47. Ross R, Ross XL, Schwing J, Längin T, Reske-Kunz AB (1998) The actinbundling protein fascin is involved in the formation of dendritic processes in maturing epidermal Langerhans cells. J Immunol 160: 3776-3782.

48. Hashimoto Y, Kim DJ, Adams JC (2011) The roles of fascins in health and disease. J Pathol 224: 289-300.

49. Chen L, Yang S, Jakoncic J, Zhang JJ, Huang XY (2010) Migrastatin analogues target fascin to block tumour metastasis. Nature 464: 1062-1066.

50. Quintana E, Shackleton M, Sabel MS, Fullen DR, Johnson TM, et al. (2008) Efficient tumour formation by single human melanoma cells. Nature 456: 593598.

51. Schatton T, Frank MH (2009) Antitumor immunity and cancer stem cells. Ann N Y Acad Sci 1176: 154-169.

52. Chin L, Garraway LA, Fisher DE (2006) Malignant melanoma: genetics and therapeutics in the genomic era. Genes Dev 20: 2149-2182.

53. Schatton T, Murphy GF, Frank NY, Yamaura K, Waaga-Gasser AM, et al. (2008) Identification of cells initiating human melanomas. Nature 451: 345-349.

54. Schatton T, Schütte U, Frank NY, Zhan Q, Hoerning A, et al. (2010) Modulation of T-cell activation by malignant melanoma initiating cells. Cancer Res 70: 697708.

55. Burger PC, Vogel FS, Green SB, Strike TA (1985) Glioblastoma multiforme and anaplastic astrocytoma. Pathologic criteria and prognostic implications. Cance 56: 1106-1111

56. Lacroix M, Abi-Said D, Fourney DR, Gokaslan ZL, Shi W, et al. (2001) A multivariate analysis of 416 patients with glioblastoma multiforme: prognosis, extent of resection, and survival. J Neurosurg 95: 190-198.

57. Chahlavi A, Rayman P, Richmond AL, Biswas K, Zhang R, et al. (2005) Glioblastomas induce T-lymphocyte death by two distinct pathways involving gangliosides and CD70. Cancer Res 65: 5428-5438.

58. Walker DG, Chuah T, Rist MJ, Pender MP (2006) T-cell apoptosis in human glioblastoma multiforme: implications for immunotherapy. J Neuroimmuno 175: 59-68.

59. El Andaloussi A, Lesniak MS (2006) An increase in CD4+CD25+FOXP3+ regulatory $\mathrm{T}$ cells in tumor-infiltrating lymphocytes of human glioblastoma multiforme. Neuro Oncol 8: 234-243.

60. Wei J, Barr J, Kong LY, Wang Y, Wu A, et al. (2010) Glioma-associated cancerinitiating cells induce immunosuppression. Clin Cancer Res 16: 461-473.

61. Wu A, Wei J, Kong LY, Wang Y, Priebe W, et al. (2010) Glioma cancer stem cells induce immunosuppressive macrophages/microglia. Neuro Oncol 12 1113-1125.

62. Wei J, Barr J, Kong LY, Wang Y, Wu A, et al. (2010) Glioblastoma cancerinitiating cells inhibit T-cell proliferation and effector responses by the signal transducers and activators of transcription 3 pathway. Mol Cancer Ther 9: 6778 .

63. Jemal A, Tiwari RC, Murray T, Ghafoor A, Samuels A, et al. (2004) Cance statistics, 2004. CA Cancer J Clin 54: 8-29.

64. Mani SA, Guo W, Liao MJ, Eaton EN, Ayyanan A, et al. (2008) The epithelialmesenchymal transition generates cells with properties of stem cells. Cell 133 : 704-715.

65. Akalay I, Janji B, Hasmim M, Noman MZ, André F, et al. (2013) Epithelialto-Mesenchymal Transition and Autophagy Induction in Breast Carcinoma Promote Escape from T-cell-Mediated Lysis. Cancer Res 73: 2418-2427.

66. Kudo-Saito C, Shirako H, Takeuchi T, Kawakami Y (2009) Cancer metastasis is accelerated through immunosuppression during Snail-induced EMT of cancer cells. Cancer Cell 15: 195-206 